\title{
MODERNE WIRTSCHAFTSBÜCHER
}

\author{
Herausgegeben von Professor Dr. Eduard Mändle
}

\author{
Allgemeine Betriebswirtschaftslehre: \\ Professor Dr. Gerhard Vogler, Fachhochschule Heilbronn \\ Kosten- und Leistungsrechnung: \\ Professor Dr. Günter Ebert, Fachhochschule Nürtingen \\ Bilanzen: \\ Professor Gerhard Kostka, Fachhochschule Nürtingen \\ Operations-Research I: \\ Professor Dr. Bodo Runzheimer, Fachhochschule für Wirtschaft Pforzheim \\ Operations-Research II: \\ Professor Dr. Bodo Runzheimer, Fachhochschule für Wirtschaft Pforzheim \\ Personalwesen: \\ Professor Dr. Fritz Bisani, Universität (GH) Essen \\ Betriebswirtschaftliche Datenverarbeitung: \\ Dr. Jürgen S. Werner, Böblingen \\ Betriebspsychologie/Betriebssoziologie: \\ Professor Dr. Clemens Heidack, Fachhochschule Düsseldorf \\ Unternehmensführung: \\ Professor Dr. Hanns Hub, Fachhochschule Nürtingen \\ Personalführung: \\ Professor Dr. Fritz Bisani, Universität (GH) Essen \\ Betriebliches Ausbildungswesen: \\ Professor Dr. Heinrich Schanz, Berufspädagogische Hochschule Stuttgart \\ Beschaffungs- und Lagerwirtschaft: \\ Professor Dr. Klaus Bichler, Fachhochschule Nürtingen \\ Absatzwirtschaft: \\ Professor Dr. Werner Bantleon/Professor Dr. Eugen Wendler/Professor Dr. Jürgen Wolff, \\ alle Fachhochschule Reutlingen \\ Betriebliche Finanzwirtschaft: \\ Professor Otto Grandi, Fachhochschule Heilbronn \\ Revisions- und Treuhandwesen: \\ Wirtschaftsprüfer Professor Dr. Andreas Csik, Stuttgart \\ Industriebetriebslehre: \\ Professor Dr. Klaus Reich, Fachhochschule für Wirtschaft Stuttgart \\ Controlling: \\ Professor Dr. Rainer Bramsemann, Fachhochschule Bielefeld \\ Betriebsorganisation: \\ Professor Egon Blum, Fachhochschule Bochum \\ Allgemeine Volkswirtschaft - Einführung und Mikroökonomik: \\ Professor Dr. Udo Müller/Dr. Hartmut Pöhlmann, Technische Universität Hannover \\ Praktische Wirtschaftspolitik: \\ Professor Dr. Eduard Mändle u.a., Fachhochschule Nürtingen \\ Geldlehre - Theorie und Politik: \\ Professor Dr. Klaus-Dieter Jakob, Fachhochschule Hagen \\ Stabilisierungspolitik: \\ Professor Dr. Horst Friedrich, Universität Düsseldorf
}


Hub • Unternehmensführung 
Moderne Wirtschaftsbücher

Herausgegeben von Prof. Dr. Eduard Mändle 
Hanns Hub

\title{
Unternehmensführung
}

\author{
2., überarbeitete Auflage
}


CIP-Titelaufnahme der Deutschen Bibliothek

Hub, Hanns:

Unternehmensführung/Hanns Hub. - 2. über-

arb. Aufl. - Wiesbaden: Gabler, 1988

(Moderne Wirtschaftsbücher)

ISBN 978-3-409-33192-0

1., Auflage 1982

2., überarbeitete Auflage 1988

Der Gabler-Verlag ist ein Unternehmen der Verlagsgruppe Bertelsmann

C) Springer Fachmedien Wiesbaden 1988

Ursprünglich erschienen bei Betriebswirtschaftlicher Verlag Dr. Th. Gabler GmbH, Wiesbaden 1988

Satz: Composer-Satz Ursula Ewert, Braunschweig

Das Werk einschließlich aller seiner Teile ist urheberrechtlich geschützt.

Jede Verwertung außerhalb der engen Grenzen des Urheberrechtsgesetzes ist ohne Zustimmung des Verlags unzulässig und strafbar. Das gilt insbesondere für Vervielfältigungen, Obersetzungen, Mikroverfilmungen und die Einspeicherung und Verarbeitung in elektronischen Systemen. 


\section{Vorwort zur ersten Auflage}

Gleichgültig, ob man auf die Tätigkeit oder auf das Studienfach abhebt, läßt sich feststellen, daß in Lehre und Praxis sehr unterschiedliche Aussagen dazu gemacht werden, was zur ,Unternehmensführung“ eigentlich alles gehört und was nicht. Die hier gegebene Antwort ist weit gefaßt: In den Rahmen der Betrachtungen werden sowohl die zielgerichtete Verhaltensbeeinflussung auf individueller Ebene als auch die Gestaltung und Steuerung des Unternehmens als Ganzes einbezogen. Die nähere Bestimmung der Inhaltsschwerpunkte sowie der Betrachtungstiefe (1. Kapitel, II.) basieren vor allem auf eigenen mehrjährigen Lehrerfahrungen mit einer gleichnamigen Vorlesung.

Hierzu empfing ich zahllose wertvolle Anregungen aus Mitgliedschaften in der „Arbeitsgemeinschaft Führungslehre an Fachhochschulen“ und in der „Deutschen Management-Gesellschaft e.V.".

Die in dieser Schrift geäußerten Gedanken können und sollen allerdings in keiner Weise als repräsentativ für diese beiden Interessengemeinschaften aufgefaßt werden. Auch sind Schwachstellen dieses Buches ausschließlich meinem Unvermögen anzulasten. Wo jedoch diese Schrift auch ,Starkstellen“ aufweisen mag, sind sie ganz sicher auch auf die tiefgehenden Fachdiskussionen in den Arbeitskreisen und Kommissionen dieser Vereinigungen zurückzuführen.

Den vielen Gesprächspartnern danke ich dafür herzlich.

Hanns Hub 


\section{Vorwort zur zweiten Auflage}

Das Echo auf die erste Auflage bestätigte die Grundkonzeption des Buches, personen- und sachbezogene Aspekte der Unternehmensführung auf der Basis einer kybernetischen Betrachtungsweise ganzheitlich in einer Darstellung zu vereinen. Aus dieser unveränderten Grundkonzeption heraus wurden für die zweite Auflage vor allem Erweiterungen zu zwei Themenkomplexen vorgenommen, denen in der aktuellen Führungsdiskussion zentrale Bedeutung zukommt. Es ist dies zum einen der Komplex „Unternehmenskultur“, womit sich der neue Abschnitt „,Systemische Ansätze“ befaßt. Eine erweiterte Darstellung erfuhr auch der Aspekt der „Strategischen Führung".

Im Interesse einer Beibehaltung des Gesamtumfanges des Buches wurde das Prinzip einer stark verdichteten Darstellungsweise auch bei den neuen Abschnitten verfolgt; einige mittlerweile verzichtbar erscheinende Abschnitte wurden eliminiert.

Das Kapitel „Organisationsentwicklung“ wurde völlig neu gestaltet und enthält unter anderem ein vom Autor entwickeltes Beratungskonzept für Organisationsentwicklungsvorhaben.

Hanns Hub 


\section{Inhalt}

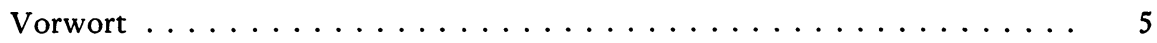

1. Kapitel: Einführung $\ldots \ldots \ldots \ldots \ldots \ldots \ldots \ldots \ldots$

I. Begriff und Erscheinungsformen der Unternebmensfübrung . . . . . 11

II. Anliegen und Aufbau des Bucbes . . . . . . . . . . . . . . . 12

III. Grundlegende Aspekte der Unternebmensfübrung . . . . . . . . . . . 14

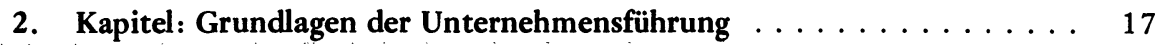

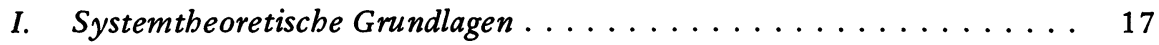

A. Der Systemcharakter des Unternehmens . . . . . . . . . . . 17

B. Die ganzheitliche Betrachtung ................ 20

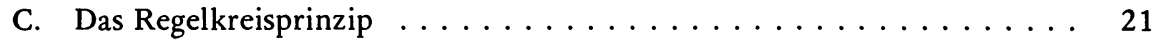

II. Allgemeine verbaltenswissenschaftliche Grundlagen . . . . . . . 27

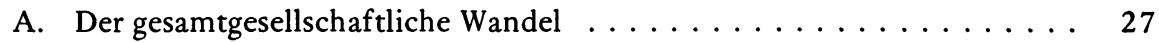

B. Die bestimmungsfaktoren des Arbeitsverhaltens . . . . . . . . . 28

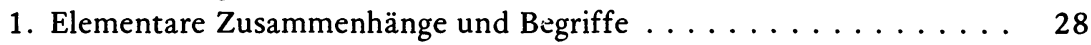

2. Ein Erklärungsschema der Wirkungen von

Verhaltensdeterminanten . . . . . . . . . . . . 30

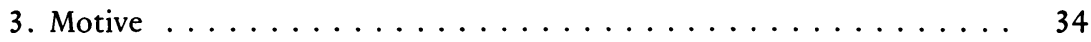

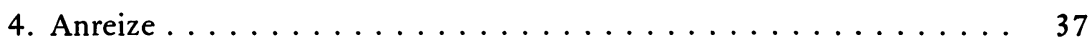

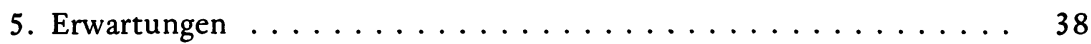

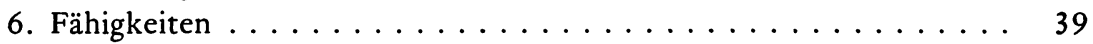

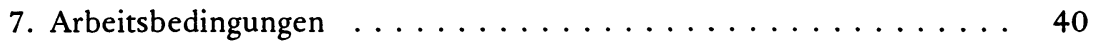

III. Ansätze zur Erklärung des Arbeitsverbaltens . . . . . . . . . 41

A. Die mechanistische Auffassung . . . . . . . . . . . . 43

B. Die sozialorientierte Auffassung $\ldots \ldots \ldots \ldots \ldots \ldots \ldots \ldots$

C. Neuere Ansätze zur Erklärung des Arbeitsverhaltens und

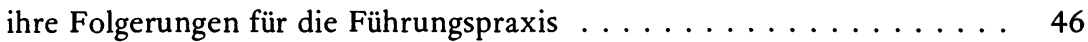

1. Die Theorie von Maslow . . . . . . . . . . . . . . . . 47

2. Die ERG-Theorie von Alderfer $\ldots \ldots \ldots \ldots \ldots \ldots \ldots \ldots \ldots$

3. Die Zwei-Faktoren-Theorie von Herzberg $\ldots \ldots \ldots \ldots \ldots$

4. Die Theorien $\mathrm{X}$ und $\mathrm{Y}$ von McGregor $\ldots \ldots \ldots \ldots \ldots$

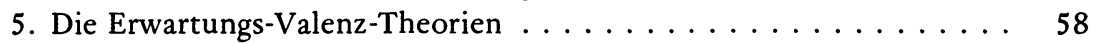

6. Die Austauschtheorie .................. 70

Literatur zum 1. und 2. Kapitel . . . . . . . . . . . 75 
3. Kapitel: Das System der Managementfunktionen $\ldots \ldots \ldots \ldots 77$

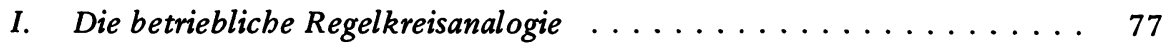

A. Erweiterungen des einfachen Regelkreises . . . . . . . . . . 78

B. Elemente und Beziehungen im erweiterten Regelkreis $\ldots \ldots \ldots \ldots 83$

II. Die Managementfunktionen im Regelkreis . . . . . . . . . 85

4. Kapitel: Die Managementfunktionen ... . . . . . . . . 93

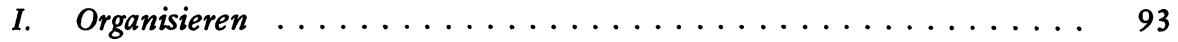

A. Grundlegende Zusammenhänge und Begriffe . . . . . . . . . . 94

B. Gliederung und Zuordnung von Aufgaben . . . . . . . . . . . 97

1. Aufgabengliederung $\ldots \ldots \ldots \ldots \ldots \ldots \ldots \ldots \ldots$

2. Institutionelle Gliederung . . . . . . . . . . . . 98

3. Aufgabenzuordnung ...................... 99

C. Beziehungsstrukturen zwischen Aufgabenträgern ........... 101

1. Hierarchische Strukturformen . . . . . . . . . . . . 101

2. Teamstrukturformen .................. 111

II. Planen und Kontrollieren . . . . . . . . . . . . . . . . . 112

A. Das System der Planungstätigkeiten . . . . . . . . . . . . 112

B. Arten der Kontrolle . . . . . . . . . . . . . . . . . 114

C. Die Strategische Führung des Unternehmens $\ldots \ldots \ldots \ldots \ldots \ldots$

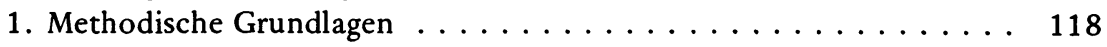

2. Empirische Grundlagen . . . . . . . . . . . . 123

3. Ubersicht der Konzepte und Instrumente . . . . . . . . . 126

4. Die Portfolio-Analyse . . . . . . . . . . . . . 128

5. Organisatorische Voraussetzungen in der strategischen

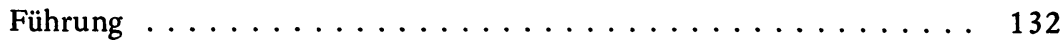

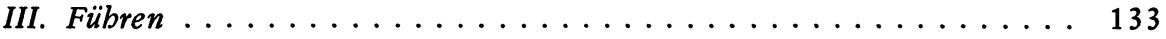

A. Grundausrichtungen von Führungstheorien $\ldots \ldots \ldots \ldots \ldots \ldots$

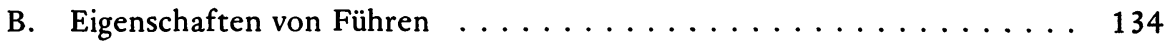

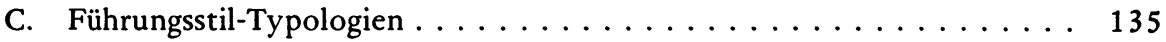

1. Arten von Führungsstilen . . . . . . . . . . . . 135

2. Das Stilkontinuum ................... 138

D. Empirische Studien zum Führungsverhalten . . . . . . . . . 140

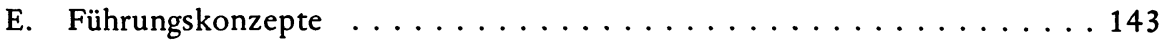

1. Das Verhaltensgitter von Blake/Mouton . . . . . . . . . 143

2. Die 3-D-Theorie von Reddin . . . . . . . . . . . . . . . . . . 144

3. Das Kontingenzmodell von Fiedler . . . . . . . . . . . . . 147 
5. Kapitel: Managementkonzeptionen $\ldots \ldots \ldots \ldots \ldots \ldots$

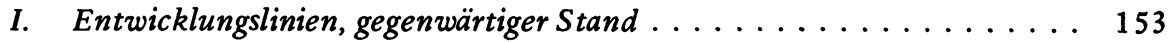

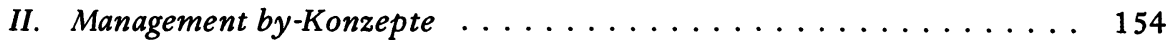

III. Managementmodelle . . . . . . . . . . . . . . . 156

A. Das Harzburger Modell . . . . . . . . . . . . . . . . . 156

B. Das Management by Objectives $(\mathrm{MbO}) \ldots \ldots \ldots \ldots \ldots \ldots \ldots \ldots$

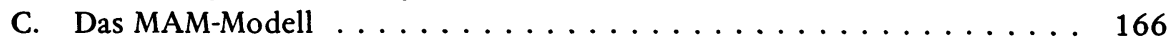

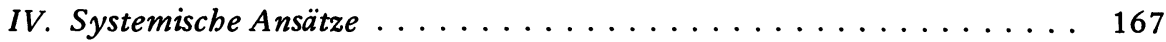

A. Das systemische Denken im Gegensatz zum analytischen Denken . . . . 168

B. Grundmerkmale einer systemischen Betrachtungsweise . . . . . . . . 169

C. Das Unternehmen als Subsystem . . . . . . . . . . . . . . . 171

D. Die Unternehmensteile als ganzheitliche Systeme . . . . . . . . . 171

E. Unternehmenskultur, Corporate Identity . . . . . . . . 172

F. Die acht Grundtugenden nach Peters/Waterman . . . . . . . . . 173

Literatur zum 3., 4. und 5. Kapitel . . . . . . . . . . . . 174

6. Kapitel: Organisationsentwicklung . . . . . . . . . . . . . . . 179

I. Begriff und Ziele der Organisationsentwicklung . . . . . . . . . . 179

II. Natürlicher Wandel von Organisationen . . . . . . . . . . . . 180

III. Modelle und Strategien der Organisationsentwicklung . . . . . . . . . 181

IV. Prozeß der Organisationsentwicklung . . . . . . . . . . . . 185

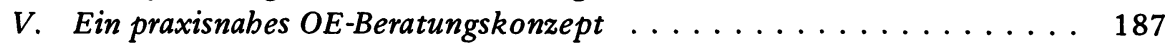

A. Rollenverteilung . . . . . . . . . . . . . . . . . . 187

B. Grundverständnis des Veränderungsprozesses . . . . . . . . . . . . . 187

C. Phasen des Veränderungsprozesses . . . . . . . . . . . . . . . 189

D. Anwendungsempfehlung . . . . . . . . . . . . . . . . . 189

Literatur zum 6. Kapitel . . . . . . . . . . . . . . . . . 189

Stichwortverzeichnis $\ldots \ldots \ldots \ldots \ldots \ldots \ldots 1$ 